\title{
Evolutionary Dynamics of Intron Size, Genome Size, and Physiological Correlates in Archosaurs
}

\section{Citation}

Waltari, Eric and Scott V. Edwards. 2002. Evolutionary dynamics of intron size, genome size, and physiological correlates in archosaurs. American Naturalist 160(5): 539-552.

\section{Published Version}

http://dx.doi.org/10.1086/342079

\section{Permanent link}

http://nrs.harvard.edu/urn-3:HUL.InstRepos:2664301

\section{Terms of Use}

This article was downloaded from Harvard University's DASH repository, and is made available under the terms and conditions applicable to Other Posted Material, as set forth at http:// nrs.harvard.edu/urn-3:HUL.InstRepos:dash.current.terms-of-use\#LAA

\section{Share Your Story}

The Harvard community has made this article openly available.

Please share how this access benefits you. Submit a story.

Accessibility 


\title{
Evolutionary Dynamics of Intron Size, Genome Size, and Physiological Correlates in Archosaurs
}

\author{
Eric Waltari ${ }^{1,2}$ and Scott V. Edwards $s^{1, *}$
}

1. Department of Zoology and the Burke Museum, University of Washington, Seattle, Washington 98195;

2. Department of Biological Sciences, Box 8007, Idaho State

University, Pocatello, Idaho 83209-8007

Submitted August 15, 2001; Accepted April 10, 2002

ABSTRACT: It has been proposed that intron and genome sizes in birds are reduced in comparison with mammals because of the metabolic demands of flight. To test this hypothesis, we examined the sizes of 14 introns in a nonflying relative of birds, the American alligator ( $\mathrm{Al}$ ligator mississippiensis), and in 19 flighted and flightless birds in 12 taxonomic orders. Our results indicate that a substantial fraction (66\%) of the reduction in intron size as well as in genome size had already occurred in nonflying archosaurs. Using phylogenetically independent contrasts, we found that the proposed inverse correlation of genome size and basal metabolic rate (BMR) is significant among amniotes and archosaurs, whereas intron and genome size variation within birds showed no significant correlation with BMR. We show statistically that the distribution of genome sizes in birds and mammals is underdispersed compared with the Brownian motion model and consistent with strong stabilizing selection; that genome size differences between vertebrate clades are overdispersed and punctuational; and that evolution of BMR and avian intron size is consistent with Brownian motion. These results suggest that the contrast between genome size/BMR and intron size/BMR correlations may be a consequence of different intensities of selection for these traits and that we should not expect changes in intron size to be significantly associated with metabolically costly behaviors such as flight.

Keywords: archosaurs, C-value, comparative method, genomics, introns, metabolic rate.

In order to explain the C-value paradox, or the lack of correlation between genome size and biological complexity, a large literature on physiological and developmental correlates of genome size has been developed (e.g., Cavalier-Smith 1985; Sessions and Larson 1987; Licht and

\footnotetext{
* Corresponding author; e-mail: sedwards@u.washington.edu.
}

Am. Nat. 2002. Vol. 160, pp. 539-552. (C) 2002 by The University of Chicago. 0003-0147/2002/16005-0001\$15.00. All rights reserved.
Lowcock 1991; Hughes and Hughes 1995; Vinogradov 1995, 1997, 1999, 2002; Monaghan and Metcalfe 2000; Gregory 2001b, 2002; Morand and Ricklefs 2001). In some of this literature, smaller genome sizes have been suggested to be the ultimate consequence of selection for smaller cell size or faster development time, or to facilitate achievement of metabolically demanding behaviors, such as flight, via smaller cell size. It is generally agreed that most of the variation in genome size among vertebrates is due to variation in the abundance of repetitive DNA species, such as SINEs, LINEs, and retrotransposons (Ohno 1970; Cavalier-Smith 1985; Pagel and Johnstone 1992). However, it has also been hypothesized that at least some of the variation can be attributed to differences in the amount of single-copy (sc) DNA and that this scDNA variation is largely due to changes in intron length (Moriyama et al. 1998; Gregory and Hebert 1999). It has been recently shown that $24 \%$ of the human genome is comprised of introns (Human Genome Consortium 2001). Wong et al. (2000) even suggested that the vast majority of "junk" DNA - comprising $97 \%$ of the human genome-could be intronic. Thus, variation in intron length could substantially modulate genome size variation. In a survey of 130 homologous introns of human and chicken, Hughes and Hughes (1995) found chicken introns to be significantly smaller than those of humans and hypothesized that the decrease in both intron and genome size in birds in comparison with mammals was in part due to the metabolic demands of flight. Supporting this hypothesis and relying primarily on the large survey of avian genome sizes published by Tiersch and Wachtel (1991), Hughes (1999) found that, among birds, stronger fliers indeed have smaller genome sizes than weaker fliers.

More recently, however, Vinogradov (1999) found no significant difference between chicken and mammalian intron sizes in a variety of analyses using a larger data set than that used by Hughes and Hughes (1995). Thus, there is no firm conclusion as to whether or not avian introns are shorter than their mammalian homologues; hence, there is no firm conclusion regarding the extent to which they are subject to selection pressures imposed by flight. 
Although studies examining correlates of genome size in vertebrates are numerous, many of these are not taxonomically comprehensive or have not been conducted using appropriate comparative methods (see Sessions and Larson 1987 or Pagel and Johnstone 1992 for exceptions). For example, Hughes and Hughes' (1995) study examined only the intron sizes of humans and chickens. Even if their data indicating that chicken introns are smaller than their human homologues were strong, this two-species comparison alone cannot be used to suggest that such decreases occurred directly or indirectly because of flight. This is because many nonflying ancestors with diverse physiologies, behaviors, and ecologies that could modulate genome size separate humans from chickens along the phylogenetic tree for vertebrates (Garland and Adolph 1994). Corroboration of the hypothesis of a link between decreases in intron size and increases in metabolic rate or flight requires the coincident evolution of these traits in the common ancestor of birds or within birds, patterns that are not yet established. Other studies, such as Vinogradov (1997), make use of comparative methods but analyze small numbers of taxa in very restricted avian clades given available information (Bennett and Harvey 1987; Tiersch and Wachtel 1991). However, Vinogradov's latest study (1999) is large but, with respect to birds, is another two-species comparison and does not employ comparative methods.

Gregory (2002), who recently confirmed an inverse correlation between resting metabolic rate and $\mathrm{C}$-value in birds, also did not use comparative methods.

Within-species, genome-wide variation in intron sizes is best understood in Drosophila, where there is a negative correlation between intron size and recombination rate. This correlation is thought to be driven by accumulation of mildly deleterious mutations and insertions in introns in genomic regions of low recombination (Carvalho and Clark 1999; Lynch 2002) or possibly by the advantages of increased recombination induced by longer introns in regions of low recombination (Comeron and Kreitman 2000). In addition, a recent study in humans and nematodes (Caenorhabditis elegans) implicates high expression levels as a selective force reducing intron length (CastilloDavis et al. 2002). The population genetic forces influencing intron length within species likely influence the among-species variance in intron length as well. However, since the database on intron length in archosaurs is so sparse, clear needs in this debate are new molecular data targeted specifically at questions of intron evolution in archosaurs. To test the hypothesis that reduced intron size in birds in comparison with mammals occurred coincidently with flight or with increases in metabolic rates, we used the polymerase chain reaction (PCR) to examine the sizes of 14 introns from 11 nuclear genes in the American alligator (Alligator mississippiensis), a nonflying member of the clade comprising the closest living relatives of birds. In addition, we have performed a comprehensive analysis of the link between metabolic rate and genomic traits as well as the evolutionary dynamics of these traits in vertebrates using recent data compilations and comparative methods. Thus, our study constitutes the first to examine in a systematic way intron size variation across archosaurs. Such surveys not only provide the foundation for robust correlative studies with other traits but also permit examination of macroevolutionary rates and trends; hence, they permit inference of the likely selection pressures, if any, that have contributed to variation in surviving lineages of birds. We emphasize that even the most basic conclusions about the evolution of vertebrate genome and intron sizes, such as whether bird and mammal genome sizes differ in a significant way, have yet to be tested using modern comparative methods. In addition, nothing is yet known about differences in rates of evolution of vertebrate genome size. Although it is still controversial whether comparative methods (sensu Felsenstein 1985) should be used at all (Ricklefs and Starck 1996), we felt it appropriate to use such methods in this study and to compare these results with those based on traditional cross-species analyses. Because there is sometimes no overlap between species for which phylogenies are known and those for which trait values are known, our willingness to assign trait values to conspecifics or congenerics in several cases, an action that we consider justified, permitted our use of comparative methods.

\section{Methods \\ Intron Amplification, Sequencing, and Sizing}

We designed 15 exon-primed intron-crossing (EPIC; Slade et al. 1993; Palumbi 1996) primer pairs for archosaurs (birds and crocodilians) using manually aligned sequences for 11 genes from human, chicken, and, in some cases, frog (Xenopus laevis) or fish (Cyprinus carpio). In addition, V. Friesen provided primer pairs for introns in the $\alpha$ tropomysin, lamin-A, and $\alpha$-enolase genes (Friesen et al. 1997, 1999), making a total of 18 primer pairs. A. Hughes kindly provided a list of introns used in Hughes and Hughes (1995), and using this list, we focused our initial PCR studies on genes with exons highly conserved between human and chicken. In all cases, primers were designed to amplify a small segment of each of the two exons flanking the intron in question so that the gene of origin of each intron could be confirmed. The sequences of primers that successfully amplified introns of the alligator and avian samples are listed in the appendix. Genomic DNA from an American alligator was isolated from blood; DNA from 19 bird species representing 11 orders was isolated 
from tissue from the Burke Museum Genetic Resources Collection or other sources using standard phenol/chloroform procedures or the QIAGEN QIAmp Tissue Kit. For the intron analyses, the following 19 bird species were studied: ostrich (Struthio camelus; Struthioniformes), elegant-crested tinamou (Eudromia elegans; Tinamiformes), dusky scrubfowl (Megapodius freycinet; Galliformes), chicken (Gallus gallus; Galliformes), green-winged teal (Anas crecca; Anseriformes), red-naped sapsucker (Sphyrapicus nuchalus; Piciformes), black cuckoo (Cuculus clamosus; Cuculiformes), budgerigar (Melopsittacus undulatus; Psittaciformes), green-breasted mountain gem ([hummingbird] Lampornus sybillae; Apodiformes), spotted owl (Strix occidentalis; Strigiformes), sharp-shinned hawk (Accipiter striatus; Falconiformes), black-footed albatross (Diomedea nigripes; Procellariformes), long-tailed mannikin (Chiroxiphia linearis; Passeriformes), Hammond's flycatcher (Empidonax hammondi; Passeriformes), Florida scrub jay (Aphelocoma coerulescens; Passeriformes), house finch (Carpodacus mexicanus; Passeriformes), redwinged blackbird (Agelaius phoeniceus; Passeriformes), brown treecreeper (Climacteris picumnus; Passeriformes), and grey-crowned babbler (Pomatostomus temporalis; Passeriformes). These species were chosen to represent phylogenetically diverse lineages encompassing the major branches of the avian tree, lineages with a wide range of published metabolic rates (e.g., passerines, hummingbird, and ratites), flightless and flighted lineages, and lineages for which there existed hypotheses for phylogenetic relationships.

Polymerase chain reactions $(25 \mu \mathrm{L})$ were carried out after determining the optimal annealing temperature for each primer pair. To determine intron sizes, we ran a HaeIII-digested $\phi$ X-174-RF DNA size ladder beside PCR products, and we plotted intron sizes on a graph of distance migrated versus log product size. For all alligator introns and a subset of the avian introns, PCR products were sequenced directly on an Applied Biosystems 373A automated sequencer using dye terminator chemistry and checked both manually and via BLAST searches to confirm identity of each intron via comparison of flanking exon sequences. In cases where PCR optimization failed to remove multiple PCR products (generally two to four products when this occurred), all products from an amplification were excised from the agarose gel, purified using the QIAGEN Gel Extraction Kit, and sequenced directly to determine the appropriate intron for analysis. The length of the conserved flanking exons was known for all introns, and these lengths were subtracted from the product sizes to determine actual intron sizes. To estimate the error in size, we used the upper and lower edge of the PCR product and routinely reran the same or reamplified products on independent gels.
Sources of Phylogenies, Genome Sizes, and Metabolic Rates

We assembled two sets of vertebrate phylogenies from a number of sources, one for analysis of intron evolution and one for analysis of genome sizes and metabolic rates. We analyzed ancestral sizes and models of archosaurian intron evolution using trees based on DNA hybridization (Sibley and Ahlquist 1990), to which we added alligator and outgroups (human, fish, or frog) using distances and divergence times from Kumar and Hedges (1998). We conducted additional analyses on an alternative topology for birds proposed by Härlid et al. (1998), but because the results were largely similar to those on the Sibley and Ahlquist (1990) tree, and because the DNA hybridization tree has recently gained support from nuclear DNA sequences (Groth and Barrowclough 1999; van Tuinen et al. 2000), we do not present them.

For analysis of genome sizes and metabolic rates across vertebrates, the topology of relationships among classes was taken from Carroll (1988), and divergence times were taken largely from Kumar and Hedges (1998). We then grafted avian relationships as in Sibley and Ahlquist (1990). As in the intron analyses, we checked the robustness of our genome size and metabolic rate results by conducting analyses using an alternative bird topology (Härlid et al. 1998) with songbirds ancestral to other avian orders. Similar results were found using either topology, and we only report the results from the Sibley and Ahlquist (1990) topology. Additional intraclass topologies and divergence times came from Cantatore et al. (1994; fishes), Feller and Hedges (1998; amphibians), Gorr et al. (1998; squamates), and Hedges and Kumar (1999; mammals). Trees for these analyses included all major tetrapod clades, including four orders of bony fish, five amphibian genera including both frogs and salamanders, one marsupial and six eutherian mammal orders, two lizards, two snakes and the tuatara, as well as one ratite bird, 19 passerine birds, and 29 species from other avian orders. Turtles were left out of our analyses (but see "Discussion") because of debate on their placement (Zardoya and Meyer 1998; Hedges and Poling 1999). The entire tree was rooted with an agnathan (lamprey). For analyses involving genome size and not metabolic rate, we analyzed data from 91 species; for bivariate analyses with metabolic rates, data from 74 species were analyzed. Mass-independent BMR data from all sources were converted to (kilocalories/day)/gram ${ }^{x}$, where $x$ is the taxonomically determined (and for birds phylogenetically determined) exponent (Withers 1992; Garland and Ives 2000). As their metabolic rates are dependent on the ambient temperature, standard metabolic rates (SMRs) of ectotherms were taken from studies in which the organism was measured at a temperature between $20^{\circ}$ and $25^{\circ} \mathrm{C}$. The major sources of these data were Bennett and 
Harvey (1987) and Vinogradov (1995). The genome size data (in units of picograms per diploid genome size) were taken principally from Tiersch and Wachtel (1991) and Vinogradov (1998). Many genome sizes were verified on the Animal Genome Size Database (Gregory 2001a). Other metabolic rate and genome size data were taken from Prosser (1973), Bennett and Dawson (1976), Olmo (1983), Lewis and Gatten (1985), Gatten et al. (1992), and Reynolds and Lee (1996). For some bird genera, metabolic rate data and genome size data were taken for congenerics rather than conspecifics. In some of these cases, the genome size used was an average of all those available for that genus. Congeneric genome data was used for the following bird genera: Penelope, Amazona, Bubo, Anthropoides, Calidris, Sterna, Leptopilos, Sphenicus, Sialia, Hirundo, Regulus, and Lonchura. This action likely did not have adverse effects on our analysis because birds have a very narrow range of genome size variation (Vinogradov 1995). In one case, genome size and metabolic rate data were taken from two different members of the family Picoides (woodpeckers). These actions were taken to produce a data set as large as possible and to enable comparative analyses. Additional details of tree topologies, branch lengths, and tip data, including their sources, can be found in the online edition of the American Naturalist.

\section{Comparative Analyses}

To test for a phylogenetic coincidence of intron and genome size reduction and the origin of flight in archosaurs, we estimated ancestral values for these traits using the computer program ANCML (Schluter et al. 1997), which calculates ancestral values of continuous traits using maximum likelihood and fully specified trees with branch lengths in millions of years as described above. Because estimates of ancestral values for continuous variables usually have extremely large errors even for large data sets (Schluter et al. 1997), we were unable to estimate ancestral intron or genome sizes with high confidence. As an alternative but statistically less desirable approach, we analyzed the raw intron data, measuring the percentage of change from human to alligator in relation to the change from human to chicken according to the equation $\left(H_{i}-A_{i}\right) /\left(H_{i}-C_{i}\right) \times 100$, where $H_{i}, A_{i}$, and $C_{i}$ are the sizes of intron $i$ (or genome size) for humans, alligators, and chicken, respectively. When intron sizes were available for multiple avian species, we used the reconstructed ancestral values for those introns on a multispecies avian tree, or we used the average sizes in place of variable $C_{i}$. Calculations using both non-log-transformed and logtransformed intron sizes were made. The log transformation was particularly important to investigate because there was evidence for a bimodal distribution of human intron sizes in this and other studies (Vinogradov 1999; fig. 1A). However, because the log transformation had no effect on the results, only the nontransformed results are shown. Some results for ancestral states were checked using Martin's (2001) COMPARE program.

All other analyses used programs from the computer package PDAP (Phenotypic Diversity Analysis Programs, version 5.0; Garland et al. 1993). To test for a correlation between mass-independent BMR/SMR and genome and intron size, we used the independent contrasts module of PDTREE (Felsenstein 1985; Garland et al. 1999). To ensure branch lengths were properly standardized before analysis, we examined the correlation between the absolute value of the standardized independent contrasts and their standard deviations for each variable, and we adjusted branch lengths to remove the correlation (Garland et al. 1992). Differences in genome size between vertebrate groups as well as deviations from a Brownian motion model for genome and intron size were examined using Monte Carlo simulations carried out in PDAP's PDSIMUL module (Garland et al. 1992). To test for differences in genome size between vertebrate groups, we used PDANOVA and PDSINGLE and the appropriate branch length transformation to compare the distribution of observed $F$ ratios for genome size differences between different pairs of clades to the distribution of 1,000 $F$ ratios obtained using simulations of genome size under a Brownian motion model (for further details, see Web site). To test for deviations from a Brownian motion model in genome size and BMR/SMR, we compared the observed coefficient of variation $(\mathrm{CV})$ of these traits in focal clades with the distribution of these values realized in 1,000 simulations under Brownian motion. Finally, we looked for differences in the evolutionary rates of genome size by comparing means of absolute values of the standardized contrasts between various pairs of clades using the independent contrasts module of PDTREE and Mann-Whitney $U$-tests (Garland 1992; Garland and Ives 2000). As above, we used branch lengths indicated by molecular divergence times estimated by Kumar and Hedges (1998). Although it has been suggested that branch length errors would have little effect on analyses such as ours (Garland et al. 1992), we also examined differences in evolutionary rates in a second analysis using divergence times estimated from the fossil record (Carroll 1988; Benton 1997).

\section{Results}

\section{Analysis of Archosaurian Intron and Genome Sizes}

We determined alligator intron sizes for 14 introns from 11 genes (fig. 1A). In five of the 14 introns, the alligator size is smaller than its chicken homologue, and among all 
A)

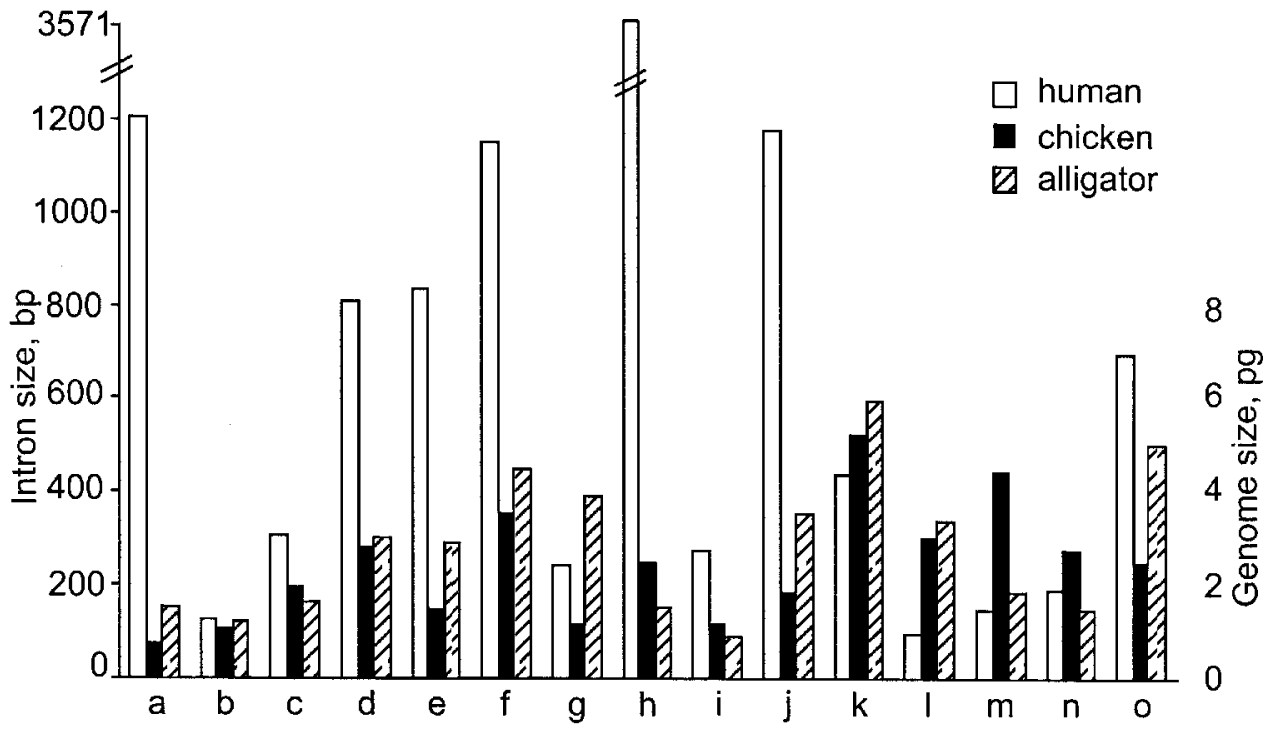

B)

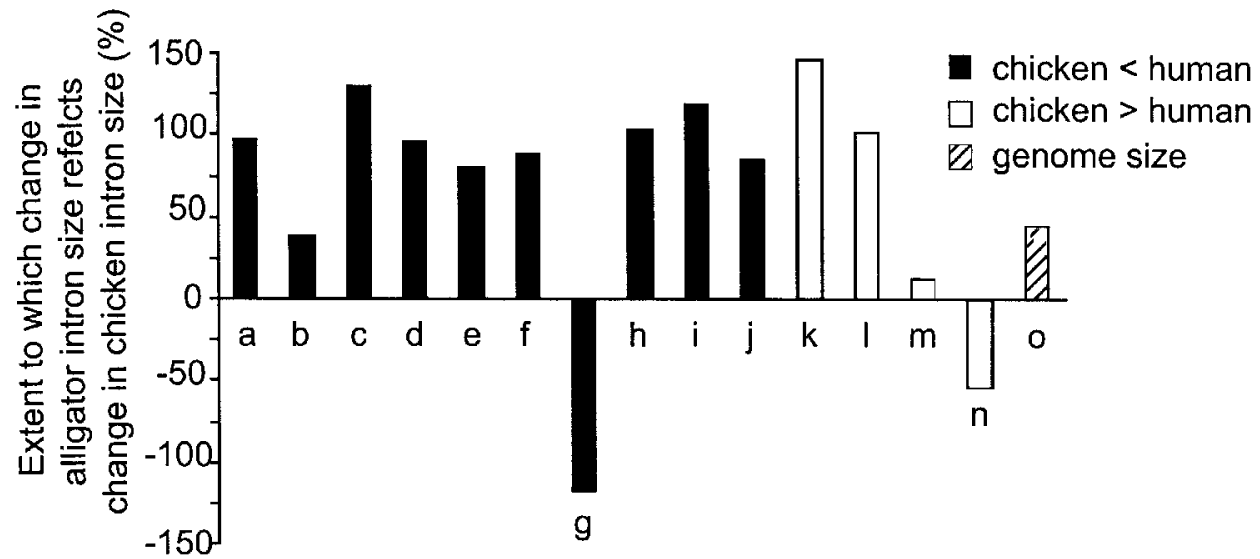

C)

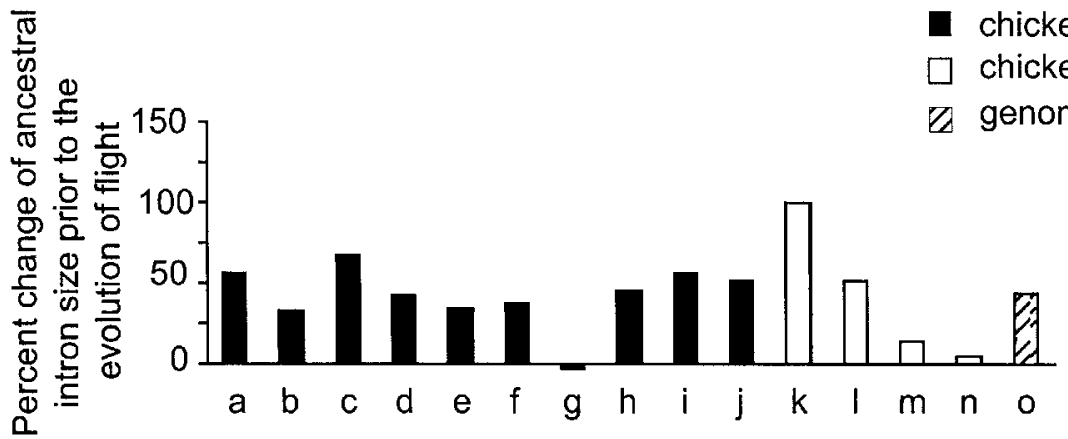

Figure 1: Trends in the evolution of 14 archosaurian intron sizes and genome size. In each panel, the 15 genomic traits analyzed are as follows: $a=$ rhodopsin (intron) 2; $b=\alpha$-cardiac actin $4 ; c=\alpha$-tubulin 3; $d=$ aldolase B $2 ; e=$ aldolase B $4 ; f=$ carbonic anhydrase II $1 ; g=$ cytosolic adenylate kinase $6 ; h=\alpha$-tropomyosin $4 ; i=\operatorname{lamin}$ A $3 ; j=\alpha$-enolase $8 ; k=\beta$-actin $2 ; l=\beta$-actin $3 ; m=\alpha$-tubulin $2 ; n=$ acetocholine receptor $\gamma$-subunit 7; $o=$ genome size. $A$, Raw data on the sizes of 14 introns in humans, chickens, and alligators; human and chicken intron sizes retrieved from GenBank; alligator intron sizes determined by polymerase chain reaction in this study. Right vertical axis applies to the last set of columns (genome size). B, Extent to which changes in alligator intron and genome size reflect the change between human and avian intron sizes, calculated as described in "Methods." A negative value indicates that the difference in size of alligator and human introns is in a direction opposite from the differences observed in comparisons of human and chicken introns. Black bars, introns for which the size in chickens is smaller than in humans; white bars, introns for which the size in chickens is larger than in humans; striped bars, genome size. $C$, Percent change of ancestral archosaurian intron size before the evolution of flight. These percentages are derived from estimates of ancestral intron sizes, calculated using the program ANCML. As the ancestral archosaur intron size becomes more similar to the avian ancestor's intron size, the percent change approaches $100 \%$. Legend is as in $B$. 
14 introns, the average human intron size is $538 \mathrm{bp}$ ( $S D=433)$, twice the average of both chicken (251 bp, $\mathrm{SD}=134)$ and alligator $(267 \mathrm{bp}, \mathrm{SD}=147)$. The size of 12 of the 14 alligator introns reflects the trend observed in comparisons of human and chicken introns to varying degrees. For the 10 introns that are smaller in chicken than in human, on average $72 \%$ of the reduction in size is observed to occur in alligators when compared with human (fig. $1 B$ ). A similar trend is observed for the four introns in our data set that are larger in chicken than in human. Approximately 52\% (fig. 1B) of the increase from human to chicken in these introns is found in comparisons of human and alligator. Overall, for both decreases and increases in intron length, an average of $66 \%$ (fig. $1 B$ ) of the trend observed in comparisons of chicken and human is also found in comparisons of alligator and human. Alligator diploid genome size $(5.0 \mathrm{pg})$ shows a similar pattern in deviating from genome size in humans $(7.0 \mathrm{pg})$ in the same direction as do chickens $(2.5 \mathrm{pg}$; fig. $1 \mathrm{~A}, 1 \mathrm{~B})$. Consistent with these patterns, we found that the correlation of the sizes of introns in chickens and alligators is significant $(r=0.54$, df $=13, .02<P<.05)$ and that, while a slope of 0 for this line was rejected, a slope of 1 was not $(b=0.603$, df $=13, P=.043)$.

\section{Genome Size Change and Correlation with Basal Metabolic Rate/Standard Metabolic Rate}

When plotted phylogenetically, the trends in genome size and BMR/SMR across vertebrates are striking. Long recognized for their very large genomes compared with amniotes (Cavalier-Smith 1985), amphibians also possess generally low SMRs, as do other ectotherms (fig. $2 A, 2 B$ ). By contrast, endotherms, particularly passerine birds on the rightmost tips of figure $2 B$, possess high BMRs. Indeed, the correlation between genome size and BMR/SMR across vertebrates without correcting for phylogeny is significant $(r=-0.488, \mathrm{df}=72, P<.001)$. However, under independent contrasts, the correlation loses significance ( $r=-0.055, \mathrm{df}=72, .5<P<.9$; branch lengths raised to the 0.75 power). Within amniotes and archosaurs individually, however, the correlation of genome size and BMR/SMR remains significant under independent contrasts (amniotes: $r=-0.315, \mathrm{df}=63, .01<P<.015$, branch lengths raised to the 0.5 power; archosaurs: $r=$ -0.368 , df $=48, .005<P<.01$, branch lengths raised to the 0.2 power). To investigate possible correlations of intron size and BMR in birds, we amplified the eight targeted introns across 19 avian taxa (fig. 3). However, we failed to find a correlation with BMR for intron size in birds. Using independent contrasts under a variety of branch length transformations, we found no significant correla- tions between any of the eight intron sizes and BMR $(r=-0.140$ to 0.349 , df $=10-12, P>.1)$.

\section{Model of Genome Size Evolution}

After comparing actual to simulated ANOVA $F$ ratios, we confirmed suggestions based on pairwise, nonphylogenetic comparisons (Tiersch and Wachtel 1991) that birds indeed have significantly smaller genome sizes than do mammals $(F=723.9 ; P<.001)$. The observed $F$ value $(85.5)$ in an ANOVA on genome size using all five major vertebrate clades in our study as variates is also significant $(P=$ $.01)$, indicating, not surprisingly, that genome size varies significantly among clades. Avian genomes were also found to be smaller than those of lepidosaurs (lizards and snakes) under the traditional avian topology $(F=27.2 ; P=$ $.066)$, but this difference is not significant probably because of low sample size within lepidosaurs.

Tests for deviations from a Brownian motion model were conducted for genome size and BMR/SMR using simulations. For genome size, the observed CV for both birds and mammals is significantly smaller than in simulations (table 1). By contrast, when all five major clades are considered together, the observed distribution in genome size is overdispersed (larger CV) when compared with simulated distributions (table 1). Basal metabolic rate/standard metabolic rate showed no significant deviations from a Brownian motion model for any analysis (table 1). Coefficients of variation of avian introns are generally consistent with a Brownian motion model (fig. 3; table 1).

\section{Rates of Evolution of Genome Size}

All comparisons of evolutionary rates involving amphibians strongly indicate that the long-term rate of change in this lineage has been higher than in other clades (table 2). In addition, using molecular divergence times (Kumar and Hedges 1998), we find that the evolutionary rate of genome size in birds is lower than in mammals or fishes, but these differences are not significant $(P=.0908$ and .0523 , respectively).

\section{Discussion}

\section{Dynamics of Genome Size Variation in Archosaurs}

The recent study of genome and intron size evolution in archosaurs has relied almost entirely on previously published data, with the result that analyses are often compromised by a lack of taxonomic comprehensiveness and a comparative approach. For example, in a comparison of data from humans and chickens only, Hughes and Hughes 


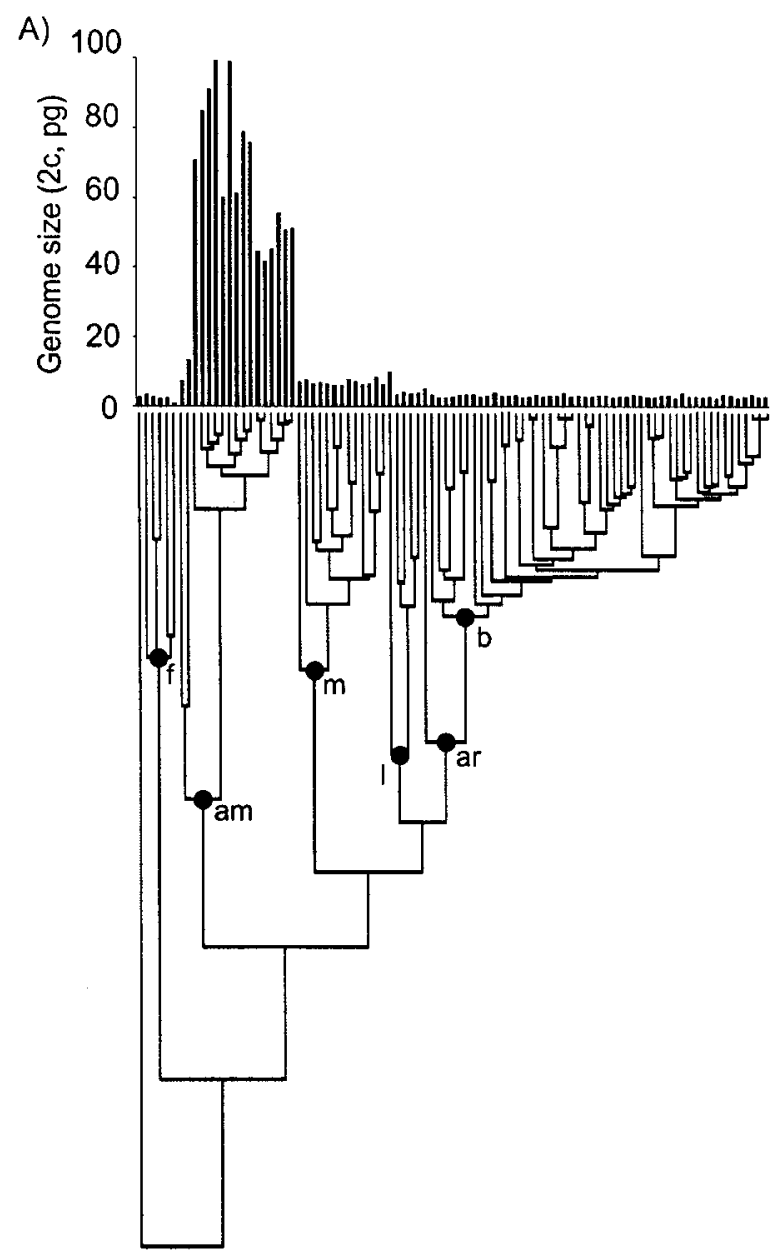

B)

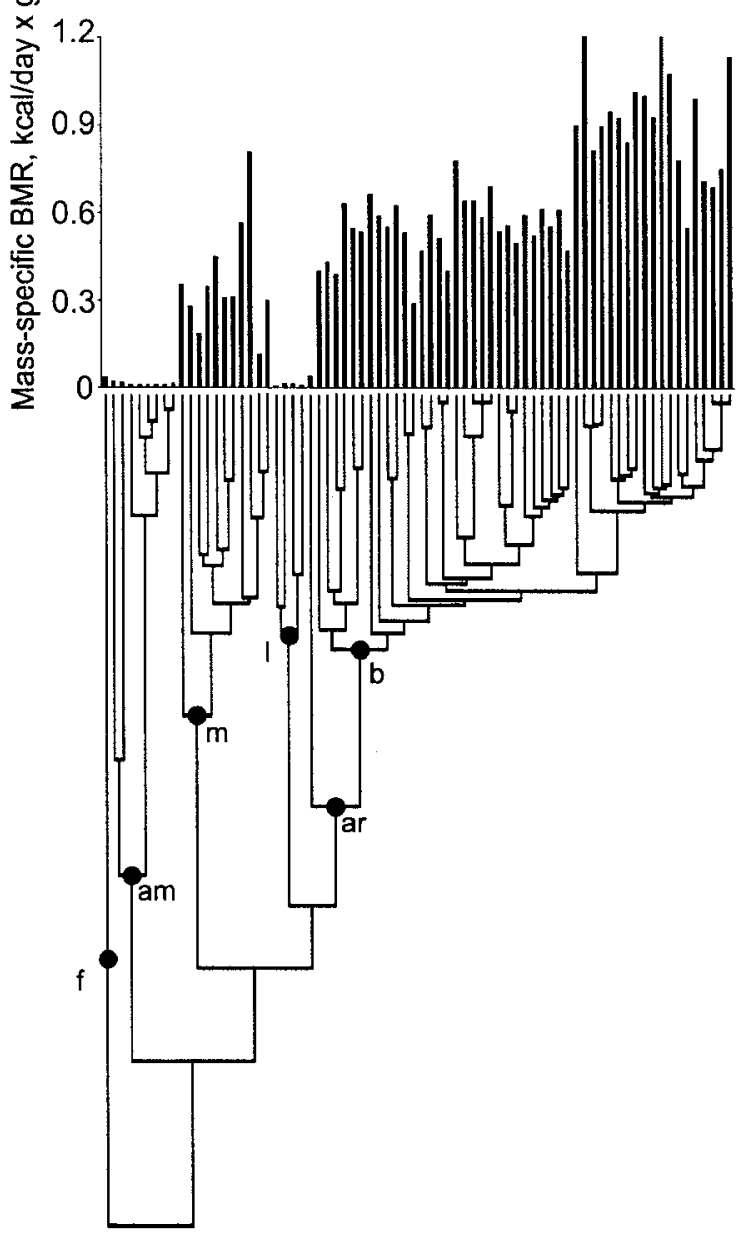

Figure 2: $A$, Phylogeny of 91 vertebrates, with a plot of diploid genome sizes measured in picograms. Letters correspond to the following clades, indicated by circles: $f=$ fish; $a m=$ amphibians; $m=$ mammals; $l=$ lepidosaurs (snakes and lizards); $a r=$ archosaurs (alligator and birds); $b=$ birds. $B$, Phylogeny of 74 vertebrates, with a plot of mass-specific BMR/SMRs measured in (kilocalories/day)/gram ${ }^{x}$ (for our analyses, masses were corrected to produce mass-independent BMR/SMRs). Letters are as in A. Dots of similar clades in the two trees may differ in height because of taxon sampling. See text and the online edition of the American Naturalist for further details.

(1995) proposed that intron and genome size are reduced in birds by the metabolic constraints of flight in birds, despite the fact that a comparison among many more species is required to draw biologically meaningful conclusions (Garland and Adolph 1994). To remedy these deficiencies, we collected new molecular data on intron sizes for a diversity of avian species and alligator and examined the hypothesis of a link between genome size, intron size, and BMR using comparative methods. Our analyses within archosaurs and other vertebrates qualify interpretations of reduced genomic traits in birds and suggest a more complex relationship between these variables. The introns of the American alligator are for the most part greater in size than their chicken homologues, as predicted by Hughes and Hughes' hypothesis (fig. 1A). However, they are usually much nearer in size to chickens than humans, suggesting that the small intron size of birds is at least partially a relict of intron size in the common ancestor of archosaurs. This trend in introns is further substantiated by the trend observed in genome size itself (see "Results"). Thus, both intron and genome size reduction in archosaurs may have begun before the origin of flight, not as a result of flight. The genome sizes of turtles ( $\sim 5.2 \mathrm{pg}$; Vinogradov 1998), although not analyzed in this study, are surprisingly close to those of alligators, consistent with recent suggestions that these taxa are phylogenetically close (Zardoya and Meyer 1998; Hedges and Poling 1999) and with our suggestion that genomic traits in lineages leading to birds experienced streamlining before the origin of flight.

In an analysis again using species as independent data 


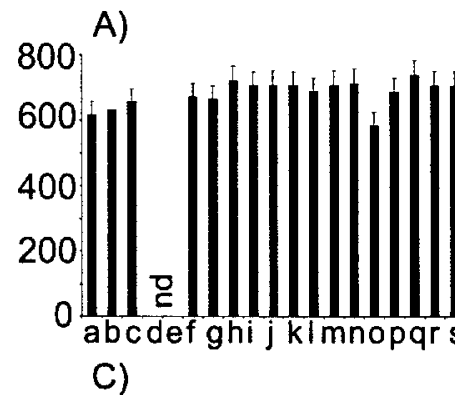

B)
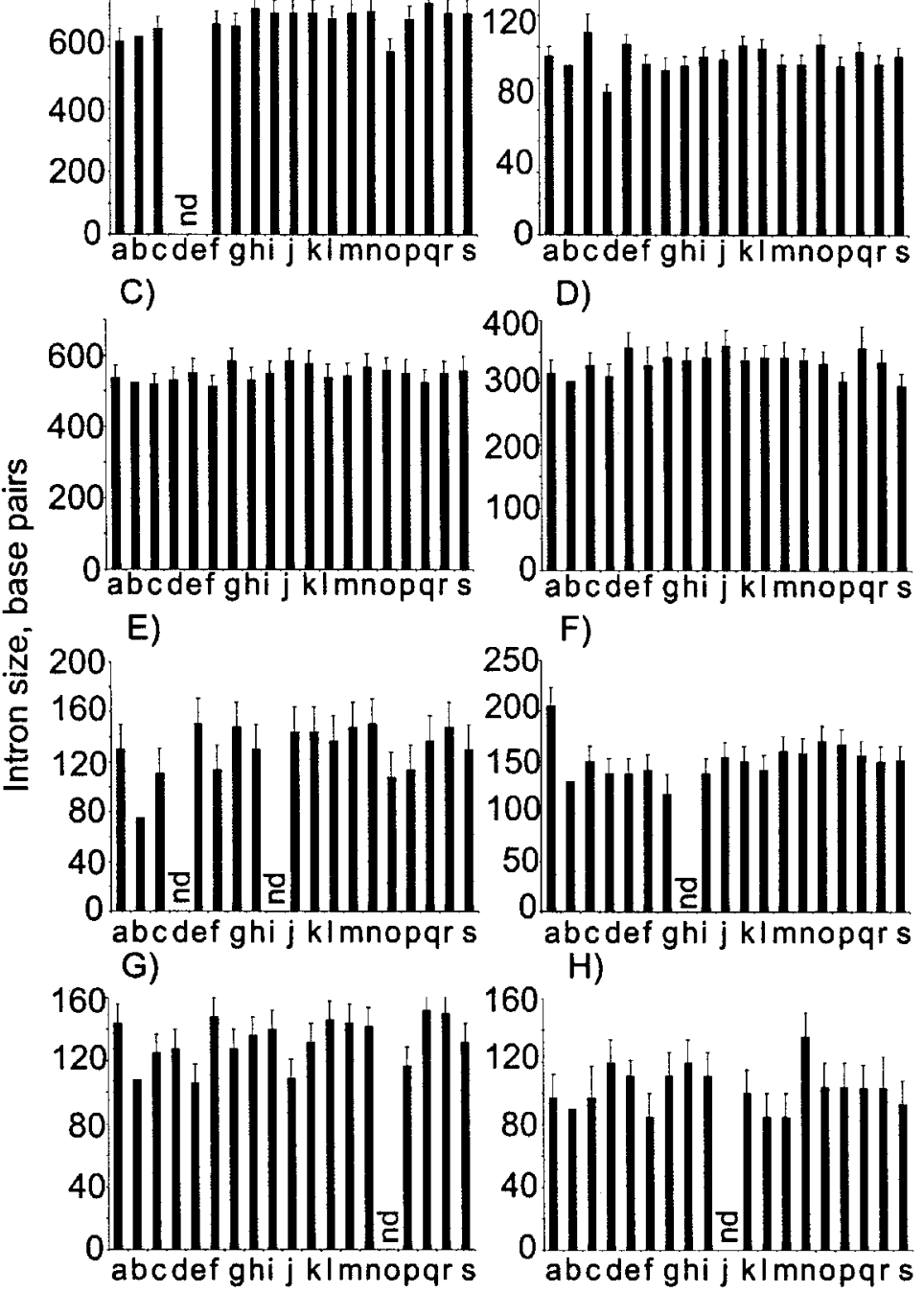

D)

400<smiles>[Li][Te]</smiles>

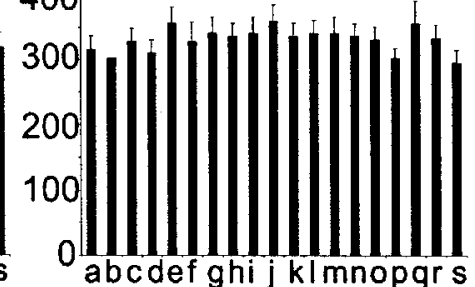

F)

250

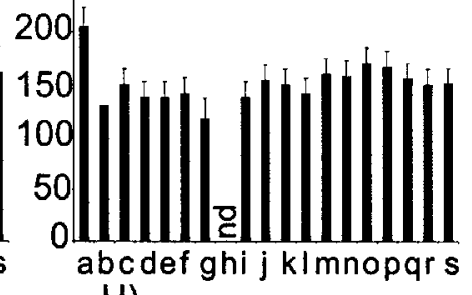

H)

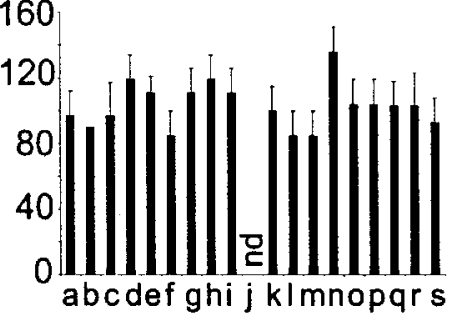

Figure 3: Survey of eight intron sizes in 19 birds. All figures shown include error bars indicating the possible intron size range (see text). Chicken intron sizes are taken from GenBank. In all figures, the species are as follows: $a=$ ostrich; $b=$ chicken; $c=$ green-winged teal; $d=$ red-naped sapsucker; $e=$ black cuckoo; $f=$ budgerigar; $g=$ green-breasted mountain gem; $h=$ spotted owl; $i=$ sharp-shinned hawk; $j=$ black-footed albatross; $k=$ Hammond's flycatcher; $l=$ Florida scrub jay; $m=$ house finch; $n=$ red-winged blackbird; $o=$ elegant-crested tinamou; $p=$ dusky scrubfowl; $q=$ brown treecreeper; $r=$ grey-crowned babbler; $s=$ long-tailed mannikin. $A, \alpha$-cardiac actin intron 3 sizes. $B$, $\alpha$-cardiac actin intron 4 sizes. $C, \beta$-actin intron 2 sizes. $D, \beta$-actin intron 3 sizes. $E$, Rhodopsin intron 2 sizes. $F$, $\alpha$-globin intron 1 sizes. $G$, $\alpha$-globin intron 2 sizes. $H, \beta$-globin intron 1 sizes.

points, Hughes (1999) found that genome size in birds is larger in lineages with reduced flight capabilities, consistent with the hypothesis of a link between genome size and BMR. When we restrict attention to variation within amniotes or archosaurs, our comparative analysis supports this view. Gregory (2002) obtained a similar result for birds examining the BMR-C-value relationship within different taxonomic categories. However, we only found a signifi- cant association between genome size and BMR for vertebrates generally when treating species as independent data points. These contradictory results likely stem from the different long-term evolutionary dynamics displayed by genome size on the one hand and BMR on the other. When viewed across the entire vertebrate tree, genome size evolution is highly punctuated, with relative stasis and low CVs within clades but dramatic differences and high 
Table 1: Tests for deviations from a Brownian motion model for variation in vertebrate genome size, mass-independent basal metabolic rate/standard metabolic rate (BMR/SMR), and avian intron size

\begin{tabular}{|c|c|c|c|c|c|}
\hline Clade/variable examined & $\begin{array}{l}\text { No. of species } \\
\text { examined }\end{array}$ & $\begin{array}{l}\text { Branch length } \\
\text { correction }\end{array}$ & $\begin{array}{l}\text { Observed } \\
\text { CV }\end{array}$ & $\begin{array}{c}\text { Mean of simulated } \\
\text { CVs }\end{array}$ & $P$ value \\
\hline \multicolumn{6}{|l|}{ Birds: } \\
\hline Genome size & 49 & Square root & .124 & 656 & $<.002^{\mathrm{a}}$ \\
\hline Mass-independent BMR & 49 & To .75 power & .327 & .459 & $.6<P<.8$ \\
\hline$\alpha$-cardiac actin intron 3 & 12 & Squared & .050 & .093 & $<.05^{\mathrm{a}}$ \\
\hline$\alpha$-cardiac actin intron 4 & 14 & To 4 th power & .081 & .074 & $.4<P<.8$ \\
\hline$\beta$-actin intron 2 & 14 & Squared & .044 & .066 & $.1<P<.2$ \\
\hline$\beta$-actin intron 3 & 14 & To 4 th power & .048 & .056 & $.6<P<.8$ \\
\hline Rhodopsin intron 2 & 12 & To 4 th power & .171 & .107 & $.1<P<.2$ \\
\hline$\alpha$-globin intron 1 & 13 & None needed & .141 & .107 & $.2<P<.4$ \\
\hline$\alpha$-globin intron 2 & 14 & Squared & .111 & .189 & $.2<P<.4$ \\
\hline$\beta$-globin intron 1 & 13 & None needed & .153 & .220 & $.4<P<.6$ \\
\hline \multicolumn{6}{|l|}{ Mammals: } \\
\hline Genome size & 13 & Square root & .114 & .696 & $<.001^{\mathrm{a}}$ \\
\hline Mass-independent BMR & 11 & To .75 power & .515 & .511 & $.8<P<1.0$ \\
\hline \multicolumn{6}{|l|}{ Amphibians: } \\
\hline Genome size & 17 & Square root & .438 & .647 & $.6<P<.8$ \\
\hline Mass-independent SMR & 8 & To .75 power & .295 & .547 & $.4<P<.6$ \\
\hline \multicolumn{6}{|l|}{ Fishes: } \\
\hline Genome size & 6 & Square root & .371 & .858 & $.1<P<.2$ \\
\hline \multicolumn{6}{|l|}{ All vertebrates: } \\
\hline Genome size & 91 & Square root & 1.751 & .750 & $<.001^{\mathrm{a}}$ \\
\hline Mass-independent BMR/SMR & 74 & To .75 power & .646 & .544 & $.6<P<.8$ \\
\hline
\end{tabular}

Note: $\mathrm{CV}=$ coefficient of variation.

a Indicates a significant difference in rate by a Mann-Whitney $U$-test.

CVs between clades. This contrasts with the pattern evident for gene number, which does not show substantial punctuation within vertebrates, or during the invertebratevertebrate transition (Martin 1999). The low CV for genome size within birds and other clades likely reflects the long-term action of stabilizing selection (Felsenstein 1988; Hansen and Martins 1996; Hansen 1997). Although some rodent genome sizes vary widely over very short evolutionary timescales (Sherwood and Patton 1982), these results largely match those of Gregory and Hebert (1999), who also noted a "saltational" pattern of genome size evolution throughout life. Single-copy gene number is likely very similar across vertebrates (Martin 1999), with differences in genome size being modulated by the origin of new classes of retroelements and by expansion and contraction of multigene families and single-copy noncoding DNA sequences. By contrast, BMR evolution appears much more labile and gradual. On the whole, we argue that it is simplistic to claim a tight correlation between genome size and BMR in vertebrates because any potential link is substantially reduced by the differences in evolutionary dynamics in the two traits.

To our knowledge, long-term rates of genome size evolution have not been investigated or compared for any clade. In general, such rates have been inferred only in- directly from the standing variation in genome size or differences in genome size between clades and not from direct comparisons of amounts of change inferred along sister lineages in a phylogeny. Despite the significant difference in genome size between birds and mammals, our analysis uncovered no evidence for a difference in evolutionary rate of genome size between these two groups. By contrast, amphibians appear to have significantly higher rates of genome size evolution than do birds, mammals, or amniotes in general. This conclusion might seem to flow inevitably from the large genome sizes or the large variation in genome size of amphibians. However, because divergence times within amphibians are relatively long, substantial variation in genome size might be expected simply by drift (Xia 1995). Like rates of point substitution in genes and proteins (Carlson et al. 1978), rapid genome size evolution in amphibians appears decoupled from rates of morphological evolution, which are almost uniformly slow.

\section{Variation in Intron Size in Archosaurs}

It may seem fruitless to search for links between variation in intron size, genome size, and BMR in archosaurs because introns presumably make up such a small fraction 
Table 2: Tests for differences in the evolutionary rate of genome size among vertebrate clades

\begin{tabular}{lcc}
\hline Pair of clades compared & $\begin{array}{c}P \text { value: molecular } \\
\text { divergence times }\end{array}$ & $\begin{array}{c}P \text { value: fossil } \\
\text { divergence times }\end{array}$ \\
\hline Birds $<$ fishes & .0523 & .3110 \\
Birds $<$ amphibians & $<.0001^{\mathrm{a}}$ & $<.0001^{\mathrm{a}}$ \\
Birds $<$ mammals & .0908 & .3507 \\
Birds $>$ lepidosaurs & .8502 & .8100 \\
Mammals $<$ fishes & .5048 & .7618 \\
Mammals $<$ amphibians & $<.0001^{\mathrm{a}}$ & $<.0001^{\mathrm{a}}$ \\
Mammals $>$ lepidosaurs & .5853 & .5048 \\
Amniotes $<$ fishes & .1314 & .4321 \\
Amniotes $<$ amphibians & $<.0001^{\mathrm{a}}$ & $<.0001^{\mathrm{a}}$ \\
\hline
\end{tabular}

Note: The direction of the inequality in the first column indicates rank of the rate of change.

${ }^{a}$ Indicates a significant difference in rate by a Mann-Whitney $U$-test.

of the archosaurian genome. However, we felt it reasonable to search for such links for several reasons. First, it has only recently been appreciated that introns make up a substantial fraction of the human (24\%), and possibly other, genomes, and one study suggests that intronic DNA may comprise the vast majority of the human genome (Wong et al. 2000). It is not known what fraction of the avian genome is comprised of introns, but it is known that the genomic fraction comprised of repetitive DNAs is much smaller in birds than in mammals. Vinogradov (1999) showed that the ratio of intragenic noncoding DNA (introns) to coding DNA is similar in chickens and mammals, suggesting that differences in genome size between these groups are largely due to intergenic DNA. It stands to reason, therefore, that introns could comprise an even larger fraction of avian genomes compared with mammalian genomes and that variation in intron length might facilitate changes in genome size, however slightly. Second, a number of recent studies suggest that mutational processes that might underlie constraints on genome size appear to be found in introns. Using variation in intron and retroelement size observed in Drosophila and crickets, Petrov et al. $(1996,2000)$ found that deletions were more common than insertions and suggested that the accumulation of such biased mutations across many singlecopy and repetitive regions could ultimately contribute to variation in genome size. Third, Vinogradov (1999) in fact detected strong correlations between genome size and intron size across eukaryotes and within mammals and vertebrates generally, although the mammal correlation was considered suspect, and none of these analyses were conducted using phylogenies. Vinogradov (1999) also found a strong correlation between intron size and BMR across vertebrates, although the single avian representative (chicken) was an outlier, having much smaller introns than its BMR would suggest. Thus, there is ample reason to investigate a possible correlation of intron and genome size and BMR in archosaurs.

To our knowledge, our survey of eight introns across a diversity of avian lineages is the first such survey of its kind for birds. We included lineages that span nearly an order of magnitude variation in BMR, from hummingbirds to ostriches. Still, we were unable to detect a correlation between intron size and BMR. This pattern is unlikely to be solely due to small sample sizes, since intron sizes tended to vary in ways not predicted by differences in genome size. For example, ostrich introns are larger than those of hummingbirds for only three of eight introns (fig. 3). Both intron size (except for the $\alpha$-cardiac actin intron 3) and BMR evolved in a manner consistent with Brownian motion, whereas genome size in birds was significantly underdispersed in birds. This might suggest that the lack of correlation of intron size and BMR does not appear to be due to contrasting modes of evolution. Still, the CVs of only three of the eight avian introns were larger than those predicted by simulations, and the CV of BMR in birds is much greater (0.459) than those for introns $(0.055-0.220)$. It is possible therefore that, like genome size, intron size in birds is underdispersed compared with BMR. Unlike genome sizes, intron sizes are unlikely to evolve punctuationally, since it is unlikely that retroelements accumulate specifically in introns as opposed to other noncoding regions, such as intergenic regions. Thus, although intron sizes appear more free to vary gradually than genome sizes, the realized range in intron sizes appears too small to reflect the influence of BMR. Further work is needed on the expected among-species variance in intron sizes given different population-genetic models of intron evolution (Carvalho and Clark 1999; Comeron and Kreitman 2000; Castillo-Davis et al. 2002; Lynch 2002).

\section{Avian Intron and Genome Sizes: Inferring Ancestral States}

Prevailing explanations for avian genome sizes assume that they comprise reductions from the state present in the common ancestor of archosaurs, or of birds and reptiles (Tiersch and Wachtel 1991). This conclusion is based primarily on the fact that avian genome sizes are small compared with those of reptiles or mammals, conclusions that were largely confirmed in this study (see also Gregory 2002). In fact, a simple reconstruction of genome sizes for common ancestors of birds, archosaurs, and amniotes, according to either a Brownian motion or a punctuated model in which change occurs at all speciation events, regardless of branch length, implies sequential stepwise reductions in the lineage leading to birds (not shown). However, such reconstructions are subject to a number of errors, particularly if the trait in question deviates from a 
Brownian motion model (Martins 1999, 2000). Given the punctuated distribution of genome size among vertebrates, the smaller size of avian genomes does not preclude a scenario in which ancestral amniote genome sizes are themselves small and undergo parallel increases in lineages leading to mammals, lepidosaurs, and archosaurs. This scenario receives some support from the fact that the genome sizes of many bony fish are similar to those of birds (Vinogradov 1998). Additionally, the rare cases, such as in amphibians, in which genome sizes of fossils can be inferred suggest that ancestral genome sizes need not be as large as those exhibited by extant lineages (Thomson 1972; Thomson and Muraszko 1978). Such increases would likely be due to parallel insertion and proliferation of retroelement families in the genomes of these lineages. There is evidence for such clade-specific, differential proliferation of repetitive elements in vertebrate genomes, such as the expansion of the chicken repeat 1 (CR1) family of non-LTR retrotransposons in birds, turtles, and reptiles (Vandergon and Reitman 1994; Kajikawa et al. 1997). The contrasting frequencies of various retroelements in different vertebrate clades suggest that once a new retroelement family becomes established in a novel genome, rapid expansion is possible or likely. Data from the human genome also suggest that, after proliferation of some retroelements, substantial elimination of these elements from specific segments of the genome can occur, resulting in the appearance of a biased pattern of retroelement insertion (Human Genome Consortium 2001). Such a scenario is tantamount to claiming that genome size does not conform to the parsimony, least squares, or maximum likelihood assumptions that underlie current models of ancestral state reconstruction for continuous characters (Martins 1999). A similar argument has been made for gene family complexity in vertebrates, which appears to have been episodic and unparsimonious (Martin 1999).

Although our PCR survey detected no clear predictors for intron size variation within birds, our comparisons of chicken, alligator, and human introns nonetheless suggest a role for history and contingency in molding intron size at higher taxonomic levels. In fact, we found that the best predictor of intron size in chickens is the size of the homologous intron in alligators. This pattern holds regardless of whether or not modern comparative methods are used. This result suggests historical contingency is at least as important in modulating intron size as natural selection or a response to factors such as BMR/SMR. For example, the sizes of chicken introns are predicted quite well from the sizes of homologous introns in alligators, and those introns that are larger in chickens than in humans are larger in alligators as well (fig. $1 A, 1 B$ ). Additionally, genome size in several clades does not appear to respond very fast to changes in BMR/SMR and is predicted better by the genome size of ancestors or sister groups. For example, lepidosaurs have generally low SMRs, yet their genome sizes are generally smaller than those of mammals (fig. 2). This suggests that genome size in lepidosaurs is at least partly a function of genome size in the common ancestor of amniotes.

Understanding of the proximate causes of genome size variation in vertebrates will reach new levels of sophistication with the advent of genome-scale sequence data for nonmammalian vertebrates (Martin 1999; Gregory $2001 b$ ). The suggestion that a large fraction of the human genome is composed of introns (Wong et al. 2000; Human Genome Consortium 2001) is just one of many surprises likely to emerge. As we have argued, this fact, in addition to the incremental effects of insertions/deletions occurring in introns throughout vertebrate genomes, suggests links between intron and genome sizes. However, the other important players in the C-value paradox as applied to vertebrates will emerge only after a similarly detailed structural understanding of diverse vertebrate genomes. For analysis of trends within archosaurs, detailed sequence information on the structure and composition of archosaur genomes and genes will be needed.

\section{Acknowledgments}

We thank T. Garland and E. Martins for technical support and for supplying the PDAP and COMPARE software packages, respectively; A. Hughes for supplying a list of introns analyzed by Hughes and Hughes (1995); and V. Friesen for additional intron primers. Alligator and ratite tissues were supplied by J. Lang and the American Museum of Natural History, respectively; all other tissues were from the Burke Museum Genetic Resources Collection. We thank D. Garrigan and J. Gasper for statistical and laboratory support; C. Laird for helpful discussion; and T. R. Gregory, R. Huey, J. Kingsolver, and two anonymous reviewers for comments that substantially improved the manuscript. We particularly thank T. R. Gregory and F. A. Kondrashov for providing access to unpublished manuscripts closely related to our study. This study was supported by National Science Foundation grants DEB9419738 and DEB-9707548 to S.V.E. 


\section{APPENDIX}

Table A1: Primers used for amplification of avian introns

\begin{tabular}{lll}
\hline Intron & \multicolumn{1}{c}{ Forward sequence } & \multicolumn{1}{c}{ Reverse sequence } \\
\hline$\alpha$-cardiac actin intron 3 & ATGACCCAGATMATGTTTGA & GTGAGGATCTTCATGAGGTA \\
$\alpha$-cardiac actin intron 4 & GAGCGTGGCTAYTCCTTTGT & GTGGCCATTTCATTCTCAAA \\
$\beta$-actin intron 2 & GATATGGAGAARATCTGGCA & ATGGGCACAGTGTGGGT \\
$\beta$-actin intron 3 & CCTGATGGTCAGGTCATCA & CAGCAATGCCAGGGTACAT \\
Rhodopsin intron 2 & TGGTGGTCTGYAAGCCCAT & TGGACCACGAACATGTAGAT \\
$\alpha$-globin intron 1 & GTCCGCTGMYGACAAGA & GGGGGAAGTAGGTYTTGGT \\
$\alpha$-globin intron 2 & CGTGGACCCDGCAACTT & CACAAGAACTTGTCCAGGG \\
$\beta$-globin intron 1 & ACTGGACTGCTGAGGAGAA & AAAGAACCTCTGGGTCCA \\
$\alpha$-tubulin intron 2 & CCCAGCGACAAGACCAT & ACTTGGTCGATGATCTCCTT \\
$\alpha$-tubulin intron 3 & CCCGAGCAACTCATCAC & AACTCAAGCTTGGACTTCTT \\
Aldolase B intron 2 & AGAMATTGCYCAGAGGATTGT & CCACMTTGATCCTCTGCA \\
Aldolase B intron 4 & WCCTCTKGCAGGAACAAA & GTCARCACCATCTTTCTTGTACT \\
Cytosolic adenylate kinase 6 & CCCGTCATCGCCTTCTA & GTCGAGGTRGGWGCAGACCT \\
Carbonic anhydrase II 1 & SSACCATGTCCCATCACT & MTTGGCRATGGGGAAGT \\
Acetocholine receptor $\gamma$-subunit 7 & CGCAAGCCGCTCTTCTA & GACAGTCTGGGCCAGGA \\
\hline
\end{tabular}

Note: Nontraditional nucleotide symbols conform to the IUPAC code.

\section{Literature Cited}

Bennett, A. F., and W. R. Dawson. 1976. Metabolism. Pages 141-149 in C. Gans and W. R. Dawson, eds. Biology of the reptilia. Academic Press, London.

Bennett, P. M., and P. H. Harvey. 1987. Active and resting metabolic rate in birds: allometry, phylogeny, and ecology. Journal of Zoology (London) 213:327-363.

Benton, M. J. 1997. Vertebrate palaeontology. Chapman \& Hall, London.

Cantatore, P., M. Roberti, G. Pesole, A. Ludovico, F. Milella, M. N. Gadaleta, and C. Saccone. 1994. Evolutionary analysis of cytochrome b sequences in some perciformes: evidence for a slower rate of evolution than in mammals. Journal of Molecular Evolution 39:589-597.

Carlson, S. S., A. C. Wilson, and R. D. Maxson. 1978. Do albumin clocks run on time? Science (Washington, D.C.) 200:1183-1185.

Carroll, R. L. 1988. Vertebrate paleontology and evolution. W. H. Freeman, New York.

Carvalho, A. B., and A. G. Clark. 1999. Intron size and natural selection. Nature 401:344.

Castillo-Davis, C. I., S. L. Mekhedov, D. L. Hartl, E. V. Koonin, and F. A. Kondrashov. 2002. Selection for short introns in highly expressed genes. Nature Genetics (in press).

Cavalier-Smith, T. 1985. The evolution of genome size. Wiley, Chichester.

Comeron, J. M., and M. Kreitman. 2000. The correlation between intron length and recombination in Drosoph- ila: dynamic equilibrium between mutational and selective forces. Genetics 156:1175-1190.

Feller, A. E., and S. B. Hedges. 1998. Molecular evidence for the early history of living amphibians. Molecular Phylogenetics and Evolution 9:509-516.

Felsenstein, J. 1985. Phylogenies and the comparative method. American Naturalist 125:1-15.

- 1988. Phylogenies and quantitative characters. Annual Review of Ecology and Systematics 19:445-471.

Friesen, V. L., B. C. Congdon, H. E. Walsh, and T. P. Birt. 1997. Intron variation in marbled murrelets detected using analyses of single strand conformational polymorphisms. Molecular Ecology 6:1047-1058.

Friesen, V. L., B. C. Congdon, M. G. Kidd, and T. P. Birt. 1999. Polymerase chain reaction (PCR) primers for the amplification of five nuclear introns in vertebrates. Molecular Ecology 8:2147-2149.

Garland, T., Jr. 1992. Rate tests for phenotypic evolution using phylogenetically independent contrasts. American Naturalist 140:509-519.

Garland, T., Jr., and S. C. Adolph. 1994. Why not to do two-species comparative studies: limitations on inferring adaptation. Physiological Zoology 67:797-828.

Garland, T., Jr., and A. R. Ives. 2000. Using the past to predict the present: confidence intervals for regression equations in phylogenetic comparative methods. American Naturalist 155:346-364.

Garland, T., Jr., P. H. Harvey, and A. R. Ives. 1992. Procedures for the analysis of comparative data using phy- 
logenetically independent contrasts. Systematic Biology 41:18-32.

Garland, T., Jr., A. W. Dickerman, C. M. Janis, and J. A. Jones. 1993. Phylogenetic analysis of covariance by computer simulation. Systematic Biology 42:265-292.

Garland, T., Jr., P. E. Midford, and A. R. Ives. 1999. An introduction to phylogenetically based statistical methods, with a new method for confidence intervals on ancestral states. American Zoologist 39:374-388.

Gatten, R. E., Jr., K. Miller, and R. J. Full. 1992. Energetics at rest and during locomotion. Pages 314-377 in M. E. Feder and W. W. Burggren, eds. Environmental physiology of the amphibians. University of Chicago Press, Chicago.

Gorr, T. A., B. K. Mable, and T. Kleinschmidt. 1998. Phylogenetic analysis of reptilian hemoglobins: trees, rates, and divergences. Journal of Molecular Evolution 47: 471-485.

Gregory, T. R. 2001a. Animal Genome Size Database. http://www.genomesize.com.

. 2001b. Coincidence, coevolution, or causation? DNA content, cell size, and the C-value enigma. Biological Reviews of the Cambridge Philosophical Society 76:65-101.

- 2002. A bird eye's view of the C-value enigma: genome size, cell size, and metabolic rate in the class Aves. Evolution 56:121-130.

Gregory, T. R., and P. D. N. Hebert. 1999. The modulation of DNA content: proximate causes and ultimate consequences. Genome Research 9:317-324.

Groth, J. G., and G. F. Barrowclough. 1999. Basal divergences in birds and the phylogenetic utility of the nuclear RAG-1 gene. Molecular Phylogenetics and Evolution 12:115-123.

Hansen, T. F. 1997. Stabilizing selection and the comparative analysis of adaptation. Evolution 51:1341-1351.

Hansen, T. F., and E. P. Martins. 1996. Translating between microevolutionary process and macroevolutionary patterns: the correlation structure of interspecific data. Evolution 50:1404-1417.

Härlid, A., A. Jarnke, and U. Arnason. 1998. The complete mitochondrial genome of Rhea americana and early avian divergences. Journal of Molecular Evolution 46: 669-679.

Hedges, S. B., and S. Kumar. 1999. Divergence times of eutherian mammals. Science (Washington, D.C.) 285: 2031a.

Hedges, S. B., and L. L. Poling. 1999. A molecular phylogeny of reptiles. Science (Washington, D.C.) 283: 998-1001.

Hughes, A. L. 1999. Adaptive evolution of genes and genomes. Oxford University Press, Oxford.
Hughes, A. L., and M. K. Hughes. 1995. Small genomes for better flyers. Nature 377:391.

Human Genome Consortium. 2001. Initial sequencing and analysis of the human genome. Nature 409:860-921.

Kajikawa, M., K. Ohshima, and N. Okada. 1997. Determination of the entire sequence of turtle CR1: the first open reading frame of the turtle CR1 element encodes a protein with a novel zinc finger motif. Molecular Biology and Evolution 14:1206-1217.

Kumar, S., and S. B. Hedges. 1998. A molecular timescale for vertebrate evolution. Nature 392:917-920.

Lewis, L. Y., and R. E. Gatten, Jr. 1985. Aerobic metabolism of American alligators, Alligator mississippiensis, under standard conditions and during voluntary activity. Comparative Biochemistry and Physiology 80A:441-447.

Licht, L. E., and L. A. Lowcock. 1991. Genome size and metabolic rate in salamanders. Comparative Biochemistry and Physiology 100B:83-92.

Lynch, M. 2002. Intron evolution as a population-genetic process. Proceedings of the National Academy of Sciences of the USA 99:6118-6123.

Martin, A. P. 1999. Increasing genomic complexity by gene duplication and the origin of vertebrates. American Naturalist 154:111-128.

Martins, E. P. 1999. Estimation of ancestral states of continuous characters: a computer simulation study. Systematic Biology 48:642-650.

- 2000. Adaptation and the comparative method. Trends in Ecology \& Evolution 15:296-299.

- 2001. COMPARE. Version 4.4. Computer programs for the statistical analysis of comparative data. http://compare.bio.indiana.edu/.

Monaghan, P., and N. B. Metcalfe. 2000. Genome size and longevity. Trends in Genetics 16:331-332.

Morand, S., and R. E. Ricklefs. 2001. Genome size, longevity and development time in birds. Trends in Genetics 17:567-568.

Moriyama, E. N., D. A. Petrov, and D. L. Hartl. 1998. Genome size and intron size in Drosophila. Molecular Biology and Evolution 15:770-773.

Ohno, S. 1970. Evolution by gene duplication. Springer, Berlin.

Olmo, E. 1983. Nucleotype and cell size in vertebrates: a review. Basic and Applied Histochemistry 27:227-256.

Pagel, M., and R. A. Johnstone. 1992. Variation across species in the size of the nuclear genome supports the junk-DNA explanation for the C-value paradox. Proceedings of the Royal Society of London B, Biological Sciences 249:119-124.

Palumbi, S. R. 1996. Nucleic acids. II. The polymerase chain reaction. Pages 205-278 in D. M. Hillis, C. Moritz, and B. K. Mable, eds. Molecular systematics. $2 \mathrm{~d}$ ed. Sinauer, Sunderland, Mass. 
Petrov, D. A., E. R. Lozovskaya, and D. L. Hartl. 1996. High intrinsic rate of DNA loss in Drosophila. Nature 384:346-349.

Petrov, D. A., T. A. Sangster, J. S. Johnston, D. L. Hartl, and K. L. Shaw. 2000. Evidence for DNA loss as a determinant of genome size. Science (Washington, D.C.) 287:1060-1062.

Prosser, C. L. 1973. Oxygen: respiration and metabolism. Pages 182-185 in C. L. Prosser, ed. Comparative animal physiology. 3d ed. Saunders, Philadelphia.

Reynolds, P. S., and R. M. Lee III. 1996. Phylogenetic analysis of avian energetics: passerines and nonpasserines do not differ. American Naturalist 147:735-759.

Ricklefs, R. E., and J. M. Starck. 1996. Applications of phylogenetically independent contrasts: a mixed progress report. Oikos 77:167-172.

Schluter, D., T. Price, A. Ø. Mooers, and D. Ludwig. 1997. Likelihood of ancestor states in adaptive radiation. Evolution 51:1699-1711.

Sessions, S. K., and A. Larson. 1987. Developmental correlates of genome size in plethodontid salamanders and their implications for genome evolution. Evolution 41: 1239-1251.

Sherwood, S. W., and J. L. Patton. 1982. Genome evolution in pocket gophers (genus Thomomys). II. Variation in cellular DNA content. Chromosoma (Berlin) 85:163-179.

Sibley, C. G., and J. E. Ahlquist. 1990. The phylogeny and classification of birds: a study in molecular evolution. Yale University Press, New Haven, Conn.

Slade, R. W., C. Moritz, A. Heideman, and P. T. Hale. 1993. Rapid assessment of single-copy nuclear DNA variation in diverse species. Molecular Ecology 2:359-373.

Thomson, K. S. 1972. An attempt to reconstruct evolutionary changes in the cellular DNA content of lungfish. Journal of Experimental Zoology 180:363-372.

Thomson, K. S., and K. Muraszko. 1978. Estimation of cell size and DNA content in fossil fishes and amphibians. Journal of Experimental Zoology 205:315-320.
Tiersch, T. R., and S. S. Wachtel. 1991. On the evolution of genome size in birds. Journal of Heredity 82:363-368.

Vandergon, T. L., and M. Reitman. 1994. Evolution of chicken repeat 1 (CR1) elements: evidence for ancient subfamilies and multiple progenitors. Molecular Biology and Evolution 11:886-898.

van Tuinen, M., C. G. Sibley, and S. B. Hedges. 2000. The early history of modern birds inferred from DNA sequences of nuclear and mitochondrial ribosomal genes. Molecular Biology and Evolution 17:451-457.

Vinogradov, A. E. 1995. Nucleotypic effect in homeotherms: body-mass-corrected basal metabolic rate of mammals is related to genome size. Evolution 49:1249-1259.

- 1997. Nucleotypic effect in homeotherms: bodymass independent resting metabolic rate of passerine birds is related to genome size. Evolution 51:220-225.

- 1998. Genome size and GC-percent in vertebrates as determined by flow cytometry: the triangular relationship. Cytometry 31:100-109.

- 1999. Intron-genome size relationship on a large evolutionary scale. Journal of Molecular Evolution 49: 376-384.

- 2002. Growth and decline of introns. Trends in Genetics 18:232-236.

Withers, P. C. 1992. Comparative animal physiology. Saunders, Fort Worth, Tex.

Wong, G. K., D. A. Passey, Y. Huang, Z. Yang, and J. Yu. 2000. Is "junk" DNA mostly intron DNA? Genome Research 10:1672-1678.

Xia, X. 1995. Body temperature, rate of biosynthesis, and evolution of genome size. Molecular Biology and Evolution 12:834-842.

Zardoya, R., and A. Meyer. 1998. Complete mitochondrial genome suggests diapsid affinities of turtles. Proceedings of the National Academy of Sciences of the USA 95: 14226-14231.

Associate Editors: Holly A. Wichman Joseph Travis 